\title{
Multicriteria decision-making method based on a cosine similarity measure between trapezoidal fuzzy numbers
}

\author{
Jun Ye \\ Department of Mechatronics Engineering, Shaoxing University, Shaoxing, Zhejiang 312000, CHINA \\ e-mail: yejnn@yahoo.com.cn, Tel +86-575-88327323
}

\begin{abstract}
The degree of similarity or dissimilarity between the objects under study plays an important role. In vector space, especially, the cosine similarity measure is often used in information retrieval, citation analysis, and automatic classification. However, it scarcely deals with trapezoidal fuzzy information and multicriteria decision-making problems. For this purpose, a cosine similarity measure between trapezoidal fuzzy numbers is proposed based on an extension of the cosine similarity between fuzzy sets and is applied to fuzzy multicriteria decision-making problems under the conditions that the criteria weights and the evaluated values in the decision matrix are expressed by the form of trapezoidal fuzzy numbers. Through the expected weight and the weighted cosine similarity measure between each alternative and the ideal alternative, the ranking order of all alternatives can be determined and the best alternative can be easily identified as well. The proposed method is simple and effective. Finally, an illustrative example demonstrates the implementation process of the technique.
\end{abstract}

Keywords: cosine similarity measure, trapezoidal fuzzy number, expected weight, multicriteria decision making

\section{Introduction}

In many real-world situations, the decision maker cannot provide deterministic alternative values but fuzzy numbers instead. This kind of uncertainty in multicriteria decision making (MCDM) can be modeled using fuzzy set theory and is ideally suited for solving these problems. Bellman and Zadeh (1970) first proposed the fuzzy decision making model. Since then, great numbers of studies have been done on fuzzy multicriteria decision making (FMCDM) (Hwang et al, 1981; Chen et al, 1992; Xu, 2004; Wang et al, 2005; Wu et al, 2007), so that the discipline has created several methodologies so far. Chen (2000) extended one of known classical MCDM method, technique for order preference by similarity to ideal solution (TOPSIS), to develop a methodology for solving multicriteria decision-making problems in fuzzy environment. Recently, Jahanshahloo et al (2006) developed the TOPSIS approach to decision making with fuzzy data, where the rating of each alternative and the weight of each criterion are expressed in triangular fuzzy numbers. He et al (2009) proposed the extension of the expected value method for multiple attribute decision making with fuzzy data, in which the preference values take the form of triangular fuzzy numbers and attribute weights are completely unknown. Moreover, Zeng (2006) developed an expected value method for FMCDM problems, in which the criteria weights and criteria values are trapezoidal fuzzy numbers.

On the other hand, Salton and McGill (1983) proposed a cosine similarity measure between fuzzy sets and applied it to information retrieval of words. Recently, Ye (2011) proposed a cosine similarity measure between intuitionistic fuzzy sets based on the concept of the cosine similarity measure between fuzzy sets and it demonstrated a stronger discrimination among the existing similarity measures by the comparisons of a variety of similarity measures for intuitionistic fuzzy sets, and then it was applied to pattern recognition and medical diagnosis. However, the domains of fuzzy sets and intuitionistic fuzzy sets are discrete sets, Trapezoidal fuzzy numbers extend discrete sets to continuous sets and are the extension of fuzzy sets. The advantage of the continuous sets is to maintain the integrity of information; while discrete sets may be loss partial information in the information integration. Therefore, the continuous sets are superior to the discrete sets. Furthermore, the existing cosine similarity measures do not deal with trapezoidal fuzzy numbers. Therefore, this paper will propose a cosine similarity measure for trapezoidal fuzzy numbers and a FMCDM method based on the cosine similarity measure under the conditions that the criterion weights and the 
evaluated values in the decision matrix are expressed by means of trapezoidal fuzzy numbers. Through the expected weight and the weighted cosine similarity measure between each alternative and the ideal alternative, the ranking order of all alternatives can be determined and the best alternative can be easily identified as well. The advantage is that the proposed FMCDM approach has some simple tools and concepts in the fuzzy similarity measure and aggregation approach among the existing ones. An illustrative example shows that the proposed method is simple and effective.

\section{Some Preliminaries}

This section introduces some definitions and basic concepts related to fuzzy sets, fuzzy numbers, and trapezoidal fuzzy numbers.

Definition 1 (Zadeh, 1965). A fuzzy set $A$ in the universe of discourse $X=\left\{x_{1}, x_{2}, \ldots, x_{n}\right\}$ is defined as follows:

$$
A=\left\{\left\langle x, \mu_{A}(x)\right\rangle \mid x \in X\right\},
$$

which is characterized by membership function $\mu_{A}(x): X \rightarrow[0,1]$, where $\mu_{A}(x)$ indicates the membership degree of the element $x$ to the set $A$.

Definition 2 (Dubois et al, 1983). Let $A$ be an fuzzy number in the set of real numbers $R$, its membership function is defined as

$$
\mu_{A}(x)=\left\{\begin{array}{l}
0, \quad x<a_{1} \\
f_{A}(x), \quad a_{1} \leq x \leq a_{2}, \\
1, \quad a_{2} \leq x \leq a_{3}, \\
g_{A}(x), \quad a_{3} \leq x \leq a_{4}, \\
0, \quad a_{4}<x .
\end{array},\right.
$$

where $a_{1}, a_{2}, a_{3}, a_{4} \in R, f_{A}:\left[a_{1}, a_{2}\right] \rightarrow[0,1]$ is a increasing continuous function, $f_{A}\left(a_{1}\right)=0, f_{A}\left(a_{2}\right)=1$, which is called the left side of the fuzzy number $A$, and $g_{A}:\left[a_{3}, a_{4}\right] \rightarrow[0,1]$ is a decreasing continuous function, $g_{A}\left(a_{3}\right)=1, g_{A}\left(a_{4}\right)=0$, which is called the right side of the fuzzy number $A$.

Particularly, if the increasing functions $f_{A}$ and decreasing functions $g_{A}$ are linear, then we have trapezoidal fuzzy numbers, which are preferred in practice. For convenience, the trapezoidal fuzzy number is usually denoted by $A=\left(a_{1}, a_{2}, a_{3}, a_{4}\right)$.

Definition 3 (Dubois et al, 1983). A trapezoidal fuzzy number $A$ with four parameters $a_{1} \leq a_{2} \leq a_{3} \leq a_{4}$ is denoted as $A=\left(a_{1}, a_{2}, a_{3}\right.$, $\left.a_{4}\right)$ in the set of real numbers $R$. In this case, its membership function can be given as

$$
\mu_{A}(x)=\left\{\begin{array}{l}
0, \quad x<a_{1} \\
\frac{x-a_{1}}{a_{2}-a_{1}}, \quad a_{1} \leq x \leq a_{2}, \\
1, \quad a_{2} \leq x \leq a_{3}, \\
\frac{x-a_{4}}{a_{3}-a_{4}}, \quad a_{3} \leq x \leq a_{4}, \\
0, \quad a_{4}<x .
\end{array},\right.
$$

The trapezoidal fuzzy number degenerates to a triangular fuzzy number when $a_{2}=a_{3}$ holds, which is considered as a special case of the trapezoidal fuzzy number.

The following properties for trapezoidal fuzzy numbers have been given by Zeng (2006).

Let $A=\left(a_{1}, a_{2}, a_{3}, a_{4}\right)$ and $B=\left(b_{1}, b_{2}, b_{3}, b_{4}\right)$ be two trapezoidal fuzzy numbers in the set of real numbers $R$ and $r$ be a positive scalar number. Then,

$$
\begin{gathered}
A+B=\left(a_{1}+b_{1}, a_{2}+b_{2}, a_{3}+b_{3}, a_{4}+b_{4}\right), \\
A-B=\left(a_{1}-b_{1}, a_{2}-b_{2}, a_{3}-b_{3}, a_{4}-b_{4}\right), \\
r A=\left(r a_{1}, r a_{2}, r a_{3}, r a_{4}\right), \\
A B=\left(a_{1} b_{1}, a_{2} b_{2}, a_{3} b_{3}, a_{4} b_{4}\right) .
\end{gathered}
$$

The expected value (Zeng, 2006) of a trapezoidal fuzzy number $A=\left(a_{1}, a_{2}, a_{3}, a_{4}\right)$ is

$$
E(A)=\frac{1}{4}\left(a_{1}+a_{2}+a_{3}+a_{4}\right) .
$$

\section{Cosine Similarity Measure between Trapezoidal Fuzzy Numbers}

In this section we introduce a definition and some concepts of the cosine similarity between fuzzy sets (Salton et al, 1983) and propose a cosine similarity measure between trapezoidal fuzzy numbers, then compare the calculation results with Chen (1996) and Chen et al (2001, 2007). 


\subsection{Cosine Similarity Measure for Fuzzy Sets}

A cosine similarity measure for fuzzy sets (Salton et al, 1983) is defined as the inner product of two vectors divided by the product of their lengths. This is nothing but the cosine of the angle between the vector representations of the two fuzzy sets.

Assume that $A=\left(\mu_{A}\left(x_{1}\right), \mu_{A}\left(x_{2}\right), \ldots, \mu_{A}\left(x_{n}\right)\right)$ and $B=\left(\mu_{B}\left(x_{1}\right), \mu_{B}\left(x_{2}\right), \ldots, \mu_{B}\left(x_{n}\right)\right)$ are two fuzzy sets in the universe of discourse $X=$ $\left\{x_{1}, x_{2}, \ldots, x_{n}\right\}, x_{i} \in X$. A cosine similarity measure (angular coefficient) between $A$ and $B$ can be defined as follows (Salton et al, 1983):

$$
C_{F}(A, B)=\frac{\sum_{i=1}^{n} \mu_{A}\left(x_{i}\right) \mu_{B}\left(x_{i}\right)}{\sqrt{\sum_{i=1}^{n} \mu_{A}^{2}\left(x_{i}\right)} \sqrt{\sum_{i=1}^{n} \mu_{B}^{2}\left(x_{i}\right)}},
$$

where $0 \leq C_{F}(A, B) \leq 1$. It is undefined if $\mu_{A}\left(x_{i}\right)=0$ and/or $\mu_{B}\left(x_{i}\right)=0(i=1,2, \ldots, n)$. Then, let the cosine measure value be zero when $\mu_{A}\left(x_{i}\right)=0$ and/or $\mu_{B}\left(x_{i}\right)=0(i=1,2, \ldots, n)$.

\subsection{Cosine Similarity Measure for Trapezoidal Fuzzy Numbers}

In this subsection, a cosine similarity measure between trapezoidal fuzzy numbers is proposed based on the concept of the cosine similarity measure for fuzzy sets.

Let $A=\left(a_{1}, a_{2}, a_{3}, a_{4}\right)$ be a trapezoidal fuzzy number in the set of real numbers $R$, the four parameters in $A$ can be considered as a vector representation with the four elements. Thus, a cosine similarity measure for trapezoidal fuzzy numbers is proposed in an analogous manner to the cosine similarity measure (angular coefficient) between fuzzy sets (Salton et al, 1983).

Assume that there are two trapezoidal fuzzy numbers $A=\left(a_{1}, a_{2}, a_{3}, a_{4}\right)$ and $B=\left(b_{1}, b_{2}, b_{3}, b_{4}\right)$ in the set of real numbers $R$. Based on the extension of the cosine similarity measure for fuzzy sets, a cosine similarity measure between $A$ and $B$ is proposed as follows:

$$
S(A, B)=\frac{\sum_{p=1}^{4} a_{p} b_{p}}{\sqrt{\sum_{p=1}^{4}\left(a_{p}\right)^{2}} \sqrt{\sum_{p=1}^{4}\left(b_{p}\right)^{2}}} .
$$

It is easy to check that the cosine similarity measure of two trapezoidal fuzzy numbers $A$ and $B$ satisfies the following properties:

P1. $0 \leq S(A, B) \leq 1$;

P2. $S(A, B)=S(B, A)$;

P3. $S(A, B)=1$ if and only if $A=B$, i.e., $a_{p}=b_{p}$ for $p=1,2,3,4$.

Proof. P1. It is obvious that the property is true according to cosine value.

P2. It is obvious that the property is true.

P3. When $A=B$, there is $a_{p}=b_{p}$ for $p=1,2,3$, 4. So there is $S(A, B)=1$. When $S(A, B)=1$, there is $a_{p}=b_{p}$ for $p=1,2,3$, 4. So there is $A=B$.

We can derive the mathematical relationship between cosine similarity and Euclidean distance when each data object has an $L_{2}$ length of 1 .

Assume that there are two trapezoidal fuzzy numbers $A=\left(a_{1}, a_{2}, a_{3}, a_{4}\right)$ and $B=\left(b_{1}, b_{2}, b_{3}, b_{4}\right)$ in the set of real numbers $R$ where each trapezoidal fuzzy number (considered as a vector) has an $L_{2}$ length of 1 . The mathematical relationship between cosine similarity and Euclidean distance is as follows:

$$
d(A, B)=\sqrt{\sum_{p=1}^{4}\left(a_{p}-b_{p}\right)^{2}}=\sqrt{\sum_{p=1}^{4}\left(a_{p}^{2}-2 a_{p} b_{p}+b_{p}^{2}\right)}=\sqrt{1-2 S(A, B)+1}=\sqrt{2[1-S(A, B)]} .
$$

\subsection{Comparison of Similarity Measures}

To illustrate the effectiveness of the proposed measure method, we use six sets of trapezoidal fuzzy numbers to compute the results of our proposed method. Then our main task is to compare the calculation results with Chen (1996) and Chen et al (2001, 2007) in this subsection.

The six sets of trapezoidal fuzzy numbers are shown in Table 1 adapted from Chen (1996) and Chen et al $(2001,2007)$, and then the calculation results of different similarity measures are shown as Table 2.

By applying our proposed method, from Table 2, we can see that the results of our proposed method coincides with ones of Chen (1996) and Chen et al. $(2001,2007)$. Therefore, the proposed method is reasonable. 
Table 1. Six sets of trapezoidal fuzzy numbers

\begin{tabular}{cc}
\hline Set numbers & Trapezoidal fuzzy numbers \\
\hline Set 1 & $A=(0.1,0.2,0.3,0.4), B=(0.1,0.25,0.25,0.4)$ \\
Set 2 & $A=(0.1,0.2,0.3,0.4), B=(0.5,0.65,0.65,0.8)$ \\
Set 3 & $A=(0.1,0.2,0.3,0.4), B=(0.3,0.45,0.45,0.6)$ \\
Set 4 & $A=(0.1,0.2,0.3,0.4), B=(0.1,0.2,0.3,0.4)$ \\
Set 5 & $A=(0.1,0.2,0.3,0.4), B=(0.5,0.6,0.7,0.8)$ \\
Set 6 & $A=(0.1,0.2,0.3,0.4), B=(0.3,0.4,0.5,0.6)$ \\
\hline
\end{tabular}

Table 2. Calculation results of different similarity measures

\begin{tabular}{ccccc}
\hline Set numbers & $\begin{array}{c}\text { Chen's method } \\
(1996)\end{array}$ & $\begin{array}{c}\text { Chen and Chen's } \\
\text { method (2001) }\end{array}$ & $\begin{array}{c}\text { Chen and Chen's } \\
\text { method (2007) }\end{array}$ & Proposed method \\
\hline Set 1 & 0.975 & 0.8357 & 0.9499 & 0.9916 \\
Set 2 & 0.6 & 0.3086 & 0.5846 & 0.9633 \\
Set 3 & 0.8 & 0.5486 & 0.7794 & 0.9774 \\
Set 4 & 1 & 1 & 1 & 1 \\
Set 5 & 0.6 & 0.36 & 0.6 & 0.9689 \\
Set 6 & 0.8 & 0.64 & 0.8 & 0.9844 \\
\hline
\end{tabular}

\section{Cosine Similarity Measure for Multicriteria Decision-Making Problems}

In this section, we present a handling method of a cosine similarity measure between trapezoidal fuzzy numbers for multicriteria decision-making problems with fuzzy weights.

Let $A=\left\{A_{1}, A_{2}, \ldots, A_{m}\right\}$ be a set of alternatives and let $C=\left\{C_{1}, C_{2}, \ldots, C_{n}\right\}$ be a set of criteria. The preference value of a criterion $C_{j}(j=1,2, \ldots, n)$ on an alternative $A_{i}(i=1,2, \ldots, m)$ is an trapezoidal fuzzy number $a_{i j}=\left(a_{i j 1}, a_{i j}, a_{i j 3}, a_{i j 4}\right)(i=1,2, \ldots, m ; j=1$, $2, \ldots, n), a_{i j 1}, a_{i j 2}, a_{i j 3}, a_{i j 4} \in R$, and $a_{i j 1} \leq a_{i j 2} \leq a_{i j 3} \leq a_{i j 4}$, which indicates the degree that the alternative $A_{i}$ satisfies the criterion $C_{j}$ given by the decision maker or expert according to some evaluated criteria. Thus we can obtain a fuzzy decision matrix $A=\left(a_{i j}\right)_{m \times n}$.

The criteria are generally incommensurate, so the criterion values need to be normalized so as to transform them into comparable values. A normalizing method has been used based on the expected value operator (Zeng, 2006). In addition, the fuzzy weight vector is normalized so that the utility value of each criterion weight belongs to $[0,1]$.

\subsection{Normalizing Fuzzy Criterion Values}

Usually, there are two categories of criteria including a benefit criterion and a cost criterion in the MCDM problem. In order to eliminate the incommensurability of the criteria in dimensionless units, each criterion value $a_{i j}(i=1,2, \ldots, m ; j=1,2, \ldots, n)$ in the matrix $A=\left(a_{i j}\right)_{m \times n}$ needs to be normalized into the corresponding comparable element in the matrix $R=\left(r_{i j}\right)_{m \times n}$ by using the following formulas (Zeng, 2006):

$$
\begin{gathered}
r_{i j}=\frac{d_{i j}}{\sqrt{\sum_{i=1}^{m}\left(E\left(d_{i j}\right)\right)^{2}}} \text { for a benefit criterion, } \\
r_{i j}=\frac{p_{j}-d_{i j}}{\sqrt{\sum_{i=1}^{m}\left(E\left(p_{j}-d_{i j}\right)\right)^{2}}} \text { for a cost criterion, }
\end{gathered}
$$

where $p_{j}=\max _{1 \leq i \leq m} \sup \left\{x_{i j} \mid \mu_{i j}\left(x_{i j}\right)>0\right\}$. Then the FMCDM addresses the problem of ranking alternatives or choosing the most desirable alternative among the finite set of alternatives based on the normalized decision matrix.

\subsection{Normalizing Fuzzy Weights by Expected Weight Values}

The weight vector of criteria for the different importance of each criterion is given as the fuzzy weight vector $\omega=\left(\omega_{1}, \omega_{2}, \ldots, \omega_{n}\right)$, in which each element is expressed by a trapezoidal fuzzy number. It is normalized into the expected weight vector $w=\left(w_{1}, w_{2}, \ldots\right.$, $w_{n}$ ) by using the following calculation: 


$$
w_{j}=\frac{E\left(\omega_{j}\right)}{\sum_{j=1}^{n} E\left(\omega_{j}\right)},
$$

where an expected weight $w_{j} \geq 0$ and $\sum_{j=1}^{n} w_{j}=1$.

\subsection{Decision-Making Method}

In multicriteria decision-making environments, the concept of ideal point has been used to help the identification of the best alternative in the decision set. Although the ideal alternative does not exist in real world, it does provide a useful theoretical construct to evaluate alternatives. Therefore, we define an ideal trapezoidal fuzzy number for a criterion in the ideal alternative $A^{*}$ as $r_{j}^{*}=(1,1,1,1)$.

Thus a weighted cosine similarity measure between an alternative $A_{i}$ and the ideal alternative $A^{*}$ represented by the trapezoidal fuzzy numbers is defined as

$$
W_{i}\left(A^{*}, A_{i}\right)=\sum_{j=1}^{n} w_{j} \frac{\sum_{p=1}^{4} r_{j p}^{*} r_{i j p}}{\sqrt{\sum_{p=1}^{4}\left(r_{j p}^{*}\right)^{2}} \sqrt{\sum_{p=1}^{4}\left(r_{i j p}\right)^{2}}},
$$

which provides the global evaluation for each alternative regarding all the criteria. From Eq. (15), the larger the value of a weighted cosine similarity measure $W_{i}$, the better the alternative $A_{i}(i=1,2, \ldots, m)$. Through the weighted cosine similarity measure between each alternative and the ideal alternative, the ranking order of all the alternatives can be determined and the best alternative can be easily identified as well.

\section{Illustrative Example}

In this section, we consider the following numerical example discussed in Zeng (2006) to illustrate the proposed method for the FMCDM problem.

A company considers five investment engineering alternatives: $A_{1}, A_{2}, A_{3}, A_{4}$, and $A_{5}$. Each alterative is evaluated from four criteria: the investment amount $\left(C_{1}\right)$, the expected net income $\left(C_{2}\right)$, the venture profit $\left(C_{3}\right)$, and the venture loss $\left(C_{4}\right)$. The fuzzy ratings of the criteria and weights are listed in Table 3.

Table 3. Ratings of criteria and weights

\begin{tabular}{ccccc}
\hline & $C_{1}\left(\$ 10^{6}\right)$ & $C_{2}\left(\$ 10^{6}\right)$ & $C_{3}\left(\$ 10^{6}\right)$ & $C_{4}\left(\$ 10^{6}\right)$ \\
\hline$A_{1}$ & $(0.40,0.45,0.55,60)$ & $(0.35,0.40,0.5,0.6)$ & $(0.32,0.42,0.5,0.6)$ & $(0.03,0.04,0.05,0.06)$ \\
$A_{2}$ & $(0.9,0.95,1.05,1.1)$ & $(0.4,0.6,0.7,0.85)$ & $(0.4,0.5,0.65,0.7)$ & $(0.05,0.1,0.15,0.2)$ \\
$A_{3}$ & $(0.4,0.45,0.55,0.6)$ & $(0.2,0.3,0.4,0.5)$ & $(0.15,0.3,0.4,0.55)$ & $(0.05,0.06,0.08,0.1)$ \\
$A_{4}$ & $(0.85,0.9,1,1.05)$ & $(0.35,0.4,0.5,0.6)$ & $(0.4,0.5,0.65,0.7)$ & $(0.04,0.08,0.15,0.2)$ \\
$A_{5}$ & $(0.55,0.6,0.7,0.75)$ & $(0.25,0.3,0.4,0.45)$ & $(0.19,0.26,0.4,0.5)$ & $(0.02,0.07,0.1,0.15)$ \\
Weights & $(0.1,0.2,0.4,0.5)$ & $(0.1,0.2,0.3,0.4)$ & $(0.01,0.05,0.1,0.21)$ & $(0.21,0.31,0.36,0.47)$ \\
\hline
\end{tabular}

The decision matrix $A=\left(a_{i j}\right)_{m \times n}$ is obtained from Table 3 , in which each element represents the suitability of an alternative versus each criterion by using a trapezoidal fuzzy number $a_{i j}=\left(a_{i j 1}, a_{i j 2}, a_{i j 3}, a_{i j 4}\right) \quad(i=1,2,3,4,5 ; j=1,2,3,4)$, as follows:

$$
A=\left[\begin{array}{cccc}
(0.40,0.45,0.55,0.60) & (0.35,0.40,0.50,0.60) & (0.32,0.42,0.50,0.60) & (0.03,0.04,0.05,0.06) \\
(0.90,0.95,1.05,1.10) & (0.40,0.60,0.70,0.85) & (0.40,0.50,0.65,0.70) & (0.05,0.10,0.15,0.20) \\
(0.40,0.45,0.55,0.60) & (0.20,0.30,0.40,0.50) & (0.15,0.30,0.40,0.55) & (0.05,0.06,0.08,0.10) \\
(0.85,0.90,1.00,1.05) & (0.35,0.40,0.50,0.60) & (0.40,0.50,0.65,0.70) & (0.04,0.08,0.15,0.20) \\
(0.55,0.60,0.70,0.75) & (0.25,0.30,0.40,0.45) & (0.19,0.26,0.40,0.50) & (0.02,0.07,0.10,0.15)
\end{array}\right] .
$$

Then the fuzzy weight vector for the four criteria is $\omega=\{(0.1,0.2,0.4,0.5),(0.1,0.2,0.3,0.4),(0.01,0.05,0.1,0.21)$, $(0.21,0.31,0.36,0.47)\}$.

In the four criteria, $C_{1}$ and $C_{4}$ are cost criteria, and then $C_{2}$ and $C_{3}$ are benefit criteria. By use of Eqs. (12) and (13) the normalized matrix is given as follows: 


$$
R=\left[\begin{array}{lllll}
(0.51,0.56,0.66,0.71) & (0.34,0.38,0.48,0.58) & (0.31,0.40,0.48,0.58) & (0.56,0.60,0.64,0.68) \\
(0.00,0.05,0.15,0.20) & (0.38,0.58,0.67,0.82) & (0.38,0.48,0.63,0.67) & (0.00,0.20,0.40,0.60) \\
(0.51,0.56,0.66,0.71) & (0.19,0.29,0.38,0.48) & (0.14,0.29,0.38,0.53) & (0.40,0.48,0.56,0.60) \\
(0.05,0.10,0.20,0.26) & (0.34,0.38,0.48,0.58) & (0.38,0.48,0.63,0.67) & (0.00,0.20,0.48,0.64) \\
(0.36,0.41,0.51,0.56) & (0.24,0.29,0.38,0.43) & (0.18,0.25,0.38,0.48) & (0.20,0.40,0.52,0.72)
\end{array}\right] .
$$

By using Eq. (14), thus we can obtain the following expected weight value for a criterion $C_{j}(j=1,2,3,4)$ : $w_{1}=0.3061, w_{2}=0.2551, w_{3}=0.0944$, and $w_{4}=0.3444$.

By applying Eq. (15), we can obtain the following values of weighted cosine similarity measures:

$W_{1}\left(A^{*}, A_{1}\right)=\mathbf{0 . 9 8 8 9}, W_{2}\left(A^{*}, A_{2}\right)=0.8554, W_{3}\left(A^{*}, A_{3}\right)=0.9740, W_{4}\left(A^{*}, A_{4}\right)=0.8870$, and $W_{5}\left(A^{*}, A_{5}\right)=0.9582$.

Therefore, the alternatives can be ranked as

$A_{1}>A_{3}>A_{5}>A_{4}>A_{2}$,

which implies that the optimal alternative is $A_{1}$ and the same as the result in Zeng (2006). This also demonstrates that the proposed method is simple and effective by the comparison of the existing one (Zeng, 2006) in the FMCDM problem.

\section{Conclusions}

In this paper, we have proposed a cosine similarity measure between two trapezoidal fuzzy numbers and investigated the method of a cosine similarity measure for the FMCDM problem, in which weight values and criterion values take the form of trapezoidal fuzzy numbers. The cosine similarity measure method has been extended for ranking alternatives, and then a practical example of the developed approach has been given to select the investment alternatives. The results show that the proposed method in this paper is simple and effective. The proposed fuzzy MCDM approach is tailored of some simple tools and concepts in the fuzzy similarity measure and aggregating approach. Furthermore, the proposed cosine similarity measure is extended to trapezoidal fuzzy numbers and trapezoidal fuzzy MCDM problems. The next research work is to develop other aggregation operations for fuzzy group decision-making problems.

\section{References}

Bellman R. and Zadeh L.A. 1970. Decision making in a fuzzy environment. Management Science, Vol. 17B, No. 4, pp. 141-164. Chen S.J. and Hwang C.L. 1992. Fuzzy Multiple Attribute Decision Making: Methods and Applications. Springer-Verlag, Berlin.

Chen S.M. 1996. New methods for subjective mental workload assessment and fuzzy risk analysis. Cybernetics and Systems, Vol. 27 , No. 5, pp. 449-472.

Chen S.J. and Chen S.M. 2001. A new method to measure the similarity between fuzzy numbers. In: Proceedings of the 10th IEEE international conference on fuzzy systems, Melbourne, Australia, pp. 208-214.

Chen S.J. and Chen S.M. 2007. Fuzzy risk analysis based on the ranking of generalized trapezoidal fuzzy numbers. Applied Intelligence, vol. 26, No. 1, pp. 1-11.

Chen C.T. 2000. Extensions of the TOPSIS for group decision-making under fuzzy environment. Fuzzy Sets and Systems, Vol. 114, pp. 1-9.

Dubois D. and Prade H. 1983. Ranking fuzzy number in the setting of possibility theory. Information Sciences, Vol. 30, pp. 183224.

He Y.Y., Wang Q. and Zhou D.Q. 2009. Extension of the expected value method for multiple attribute decision making with fuzzy data. Knowledge-Based Systems, Vol. 22, pp. 63-66.

Hwang C.L. and Yoon K. 1981. Multiple Attribute Decision Making: Methods and Applications. Springer-Verlag, Berlin.

Jahanshahloo G.R., Hosseinzadeh Lotfi F. and Izadikhah M. 2006. Extension of the TOPSIS method for decision-making with fuzzy data. Applied Mathematics and Computation, Vol. 181, pp. 1544-1551.

Salton G. and McGill M.J. 1983. Introduction to modern information retrieval. McGraw-Hill, Auckland.

Wang Y.M. and Parkan C. 2005. Multiple attribute decision making based on fuzzy preference information on alternatives: ranking and weighting. Fuzzy Sets and Systems, Vol. 153, pp. 331-346.

Wu Z.B. and Chen Y.H. 2007. The maximizing deviation method for group multiple attribute decision making under linguistic environment. Fuzzy Sets and Systems, Vol. 158, pp. 1608-1617.

Xu Z.S. 2007. A method for multiple attribute decision making with incomplete weight information in linguistic setting. Knowledge-Based Systems, Vol. 20, pp. 719-725.

Xu Z.S. 2004. Uncertain Multiple Attribute Decision Making: Methods and Applications. Tsinghua University Press, Beijing.

Ye J. 2011. Cosine similarity measures for intuitionistic fuzzy sets and their applications. Mathematical and Computer Modelling, Vol. 53, No. 1-2, pp. 91-97.

Zadeh L.A. 1965. Fuzzy sets. Information Control, Vol. 8, pp. 338-353. 
Zeng L. 2006. Expected value method for fuzzy multiple attribute decision making. Tsinghua Science and Technology, Vol. 11, No. 1, pp. 102-106.

\section{Biographical note}

Jun Ye is a Professor in the Department of Mechatronics Engineering, Shaoxing University, China. He has more than 30 years of experience in teaching and research. His current area of research includes Multi-criteria Decision-Making, Soft Computing, Robotics and Automation, Intelligent Control, Pattern Recognitions, Fault Diagnosis, and Mechanical Design. He has published more than ninety papers in journals. He has written few books related to his research work. He has finished a few projects sponsored by government of China.

Received March 2011

Accepted May 2011

Final acceptance in revised form June 2011 